\title{
Parameterization of Indian Traditional Rangoli Design Patterns
}

\author{
Abhimanu Chauhan \\ Ganpati Institute of Technology \\ \& Managment \\ Bilaspur, Haryana - India
}

\author{
Kulwant Singh \\ GTB Khalsa Institute of \\ Engineering \& Tech. \\ Malout, Punjab - India
}

\author{
Arvind Kumar \\ Ganpati Institute of Technology \\ \& Managment \\ Bilaspur, Haryana - India
}

\begin{abstract}
The work presents a parametric CAD tool to offers the possibility to create the Indian traditional rangoli design patterns with the use of computer applications. To invent automation in rangoli design, this work approach towards parameterization of the rangoli design patterns belonging to Indian culture. The rangoli designs can be drawn with the application of CAD tool and further made with robotic machines. This idea plays a vital role to preserve the culture heritage. The CAD tool provides flexibility to draw the design according to available size/space. It provides inbuilt library for rangoli design of different area.
\end{abstract}

\section{Keywords}

Computer Supported Design, Digital Design, Parametric Design, Design Automation, Rangoli

\section{INTRODUCTION}

Rangoli is an art of making beautiful floor designs. The rangoli provides an object for fascination or effortless attention in the sacred space, in an atmosphere without any distractions, which results in relaxation and directed attention. The eye pursues the commonsense pattern of the rangoli. It is filled with culturally recognizable symbols for relieve, protection, and wish fulfillment, and is also a visual encounter with an aesthetically pleasing, moderately complex pattern with a focal point. Gazing on the rangoli in the sacred space can provide restorative process within the domestic lodgings [5]. India is very famous for its rich culture and traditions [2]. Here, each state even has its own language, cuisine, festivals, culture and traditions. Rangoli is one of such tradition. It is very popular in the almost Indian states. Some traditional rangoli designs are shown in figure 1 .

Traditional representational rangoli motifs were plant life such as flowers, leaves, coconut, lotus, mango, and ashwath (peepal leaf). Animals represented were cows, elephants, horses, parrots and swans. The Girls copied patterns from their mothers and grandmothers, and challenged each other for speed and precision in creating complex patterns. These days, girls copy from rangoli pattern books or weekly magazines and websites that feature new rangoli patterns featuring non-traditional patterns. Special rangoli designs are created for holidays in the Hindu religious calendar, more elaborate than the daily rangoli. 'Diwali' is the most lavish Hindu festival, occurring in October or November at the close of the rainy season, observed to propitiate the 'Lakshmi', goddess of pleasure, plenty, luck and prosperity. Hexagons and six pointed stars, and six petalled lotuses are particularly used for 'Diwali' to honor 'Lakshmi'. 'Makara Sankranti' is a winter solstice holiday. 'Kunda' patterns, circular patterns symbolizing the sun disc, are created for winter solstice. 'Holi', the festival of colours, is the second largest annual Hindu religious festival, celebrated in March.
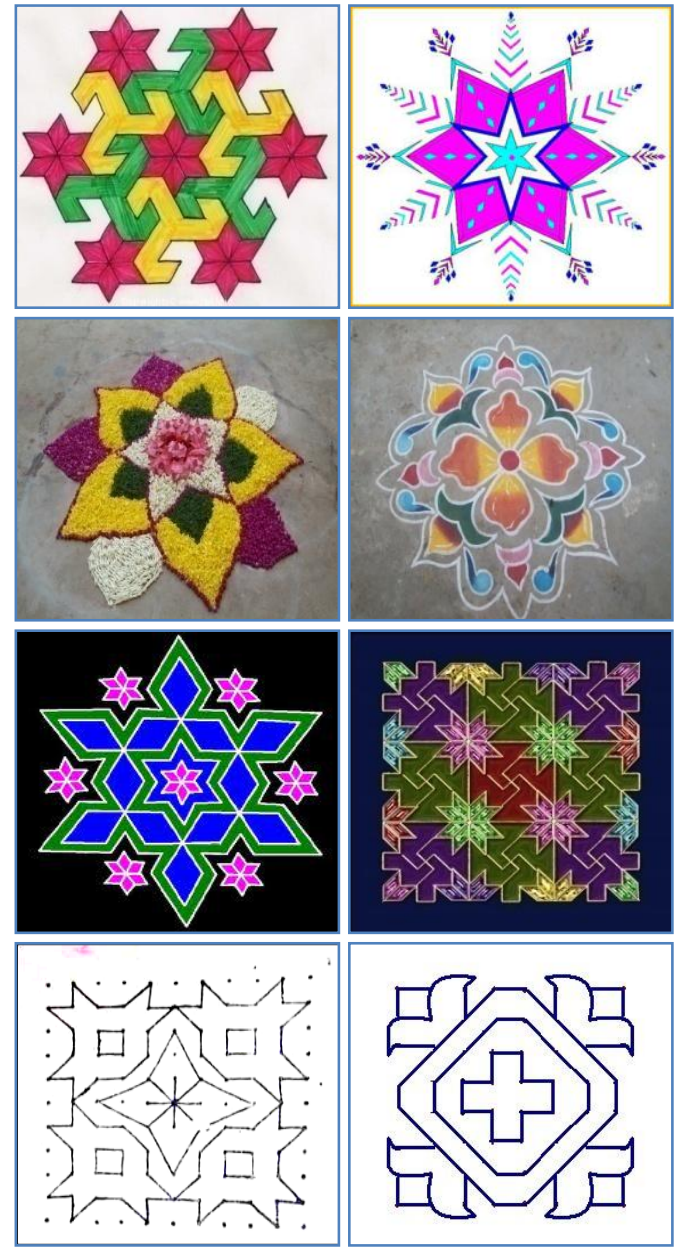

Fig. 1: The Traditional Rangoli Design Patterns

Colored rangoli powders are sold in the market to create multicolored patterns. A theme of the festival is the triumph of good over evil, and some holiday rangoli patterns feature depictions of weapons and symbols of valor. 'Gangour' is a festival to honour Shiva, the male deity whom women worship in the expectation of receiving children, luck, luxury, and riches[12]. The various special designs of rangoli making with the special theme, symbolic presentation and on special occasions are shown in figure 2. 


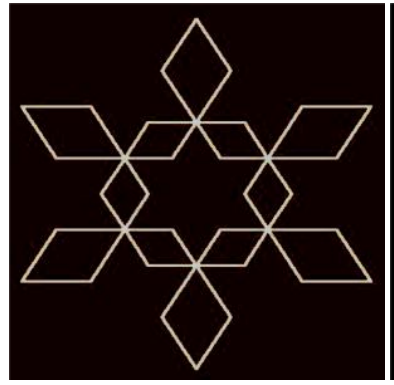

(a)

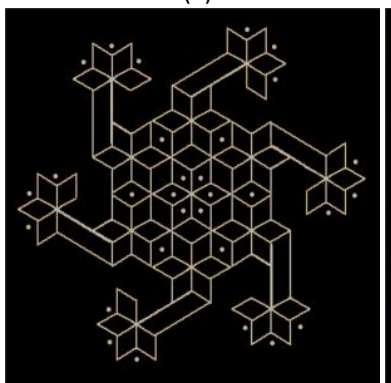

(e)

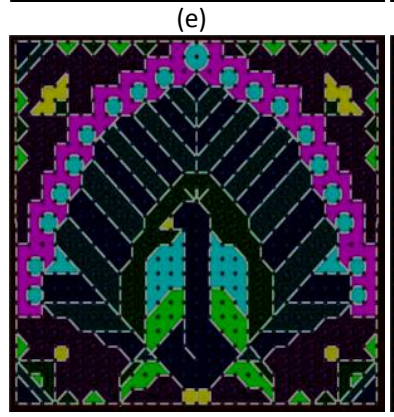

(i)

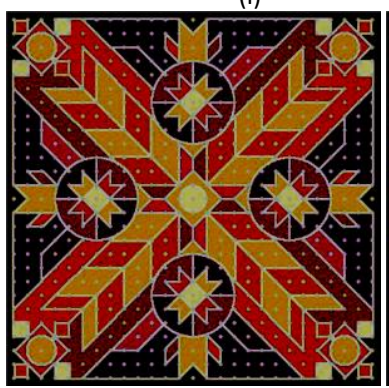

(m)

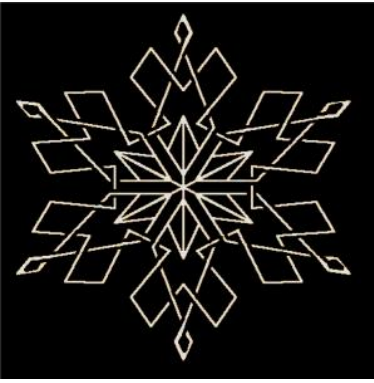

(b)

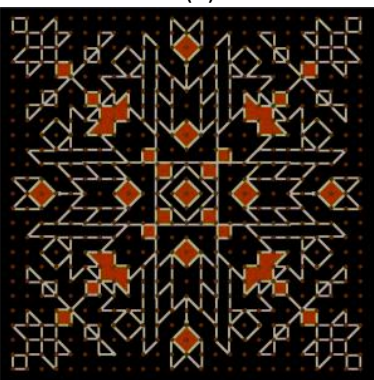

(f)

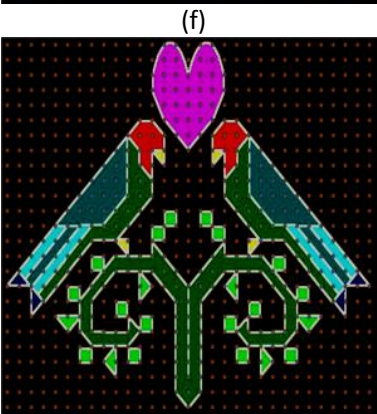

(j)

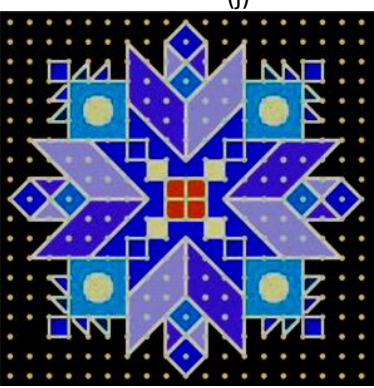

(n)

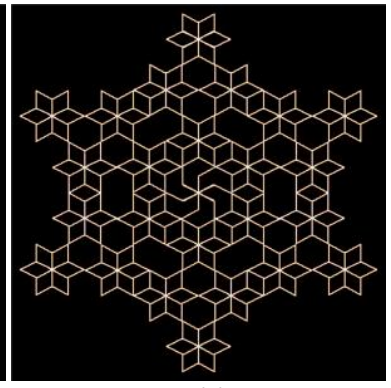

(c)

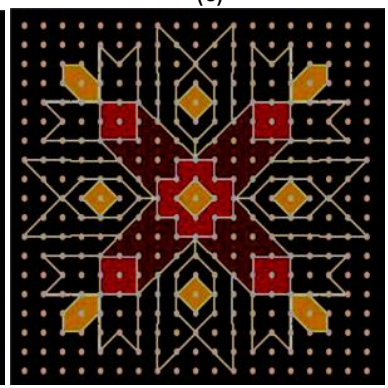

(g)

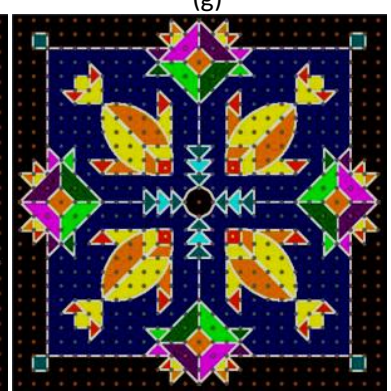

(k)

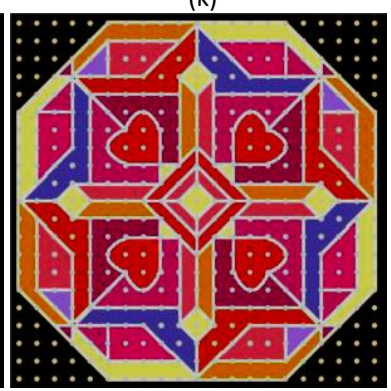

(o)

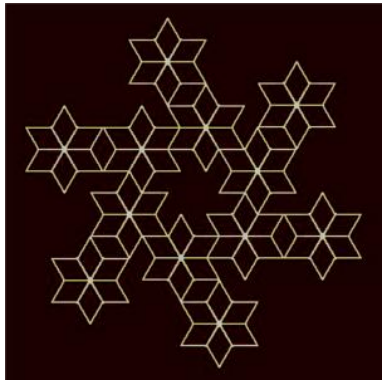

(d)

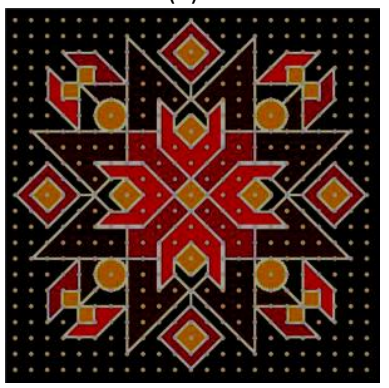

(h)
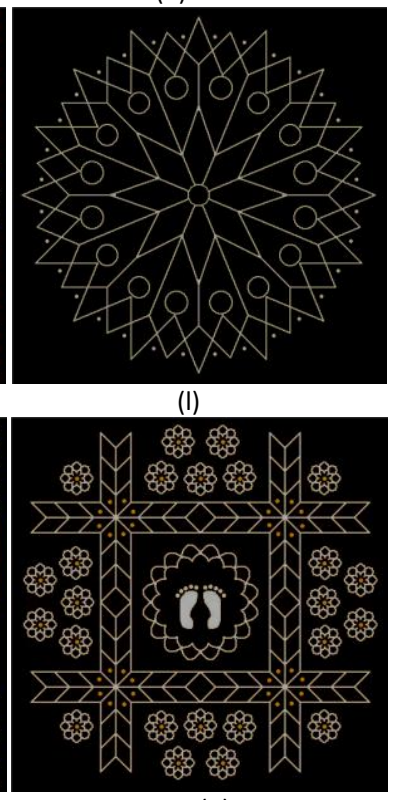

(p)

Fig. 2: (a) A simple pattern for luck; (b) Six petalled flower patterns, 'Adhakatya Phul', are drawn for the festival of lights, Diwali; (c, d, e) In Tamil Nadu,' Pulli Kolam' patterns based on 6-pointed star are drawn; (f ,g, h) In Kerala, rangoli made by laying out a grid of dots, then connecting the dots with rice flour lines and these patterns filled with colorful herbs and spices; $(i, j, k)$ A grid of dots drawn on the floor, and then connecting the dots with rice flour lines and fills the resulting pattern with different bright colored rangoli powder; (I) 'Amiyal' are lotus patterns from Kerala; (m, n, o) Kerala rangoli filled with small

flowers or flower petals for special occasions. These rangoli drawn very large for a wedding, filled with colorful fragrant

flowers and tea lights, and meant to depict a celestial garden. (p) Madhya Pradesh, the Goddess Lakshami's feet drawn in Rangoli to invoke her presence in a sacred place [7\&12].

Rangoli serves social needs as well as metaphysical needs for a Hindu family. Every day, the front door of House has fresh rangoli morning and is swept clean evening is a home where religious duties are being properly enacted, evidencing internal orderliness, piety and self-respect [9-11].

Rangoli designs are well suited to 'kaccha' houses, village homes made of mud brick that had to be resurfaced regularly; for every event, the walls and floors were renewed, and decorated with making rangoli design. As people move to city apartment blocks, rangoli has become increasingly scarce, as apartment managers refuse to return cleaning deposits when floors and walls are marked with artwork. Some premade stick-down vinyl rangoli designs are available for citydwellers who want festive adornment for holidays, but who no longer have the means or space to create traditional rangoli. Beside these obstacles, there are many problems arising due to its complex tradition of making with hand, which is a time consuming task, requires little skill and interest/hobby in the rangoli making. Therefore, its traditional making is currently fading because new upcoming generations are not interested in. Moreover, there is no indigenous knowledge and skill of 
forebears to prolong these rangoli designs. There is a fear to lose the heritage style of rangoli designs, as the new generation is not showing their interest in the traditional design and follows non-traditional patterns because of simplicity and easy availability in books or weekly magazines and websites. Consequently, this work explores different traditional rangoli design patterns. An algorithm is presented to generate the geometric patterns using the parameterized element of the design. This algorithm produces traditional style of design/patterns. Here, the objective is to invent automation in rangoli and also to enable with flexibility to variation in size according to availability of space and our choice without any horror of losing the culture legacy of the particular design. In this work, the proposed tool makes possible to produce the traditional $2 \mathrm{D}$ rangoli designs.

\section{GEOMETRIC MODELING OF MOTIFS/DESIGNS}

The representation is vitally important, because the process of design pattern illustration can be regarded as a search for feasible descriptions that the computer formulates from given input data. Firstly, the representation should describe the particular concept appropriately and it should have the ability to correspond to implicit as well as explicit information. Secondly, it should have a well-organized form so that it can execute inferences correctly with predefined knowledge. Thirdly, from the result of pattern representations, new objects should be able to be predicted or generalized. Such patterns are defined in terms of abstract entities called motifs which are neither overlapped nor separated [1]. The final rangoli design patterns are based on the repetition of the motifs positioned at regular, or measured, intervals. While generating a design, it is necessary to have a realization that how more complex design can be created from simpler shapes. In the construction of a design for particular rangoli, the design is analyzed by constructing a hierarchical representation of the design [2]. By this approach a small part of the design is created and further geometrical transformation applied to construct the complete design. The use of geometry is important in the development of the style of designs, whatever forms it takes. Circles, for example, are essential in designing floral type patterns [3]. The use of purely geometric elements to create elaborate rangoli patterns, however, has become a complicated form of beautification on its own. The appeal of geometric decoration lies in its logical interrelation of parts, reflecting in abstract form the underlying order as found in nature. The floral motifs are constructed by the combination of circles, arcs and lines [4]. Such motifs of the designs which are initially geometrically constructed as shown in figure in table 1. Taking $\mathrm{O}$ is the center point and draw two circles of radius $r \& R(r<R)$. From point A on the outer circle draw an arc of radius $R$ which gives point $B$ on circumference of outer circle. Similarly draw an arc of same radius from point A taking center point $\mathrm{B}$ which intersects the first arc at point $\mathrm{C}$. Line OA and $\mathrm{OB}$ give points $\mathrm{I}$ and $\mathrm{J}$ on inner circle. Join the points AJ and BI. The rest of motif followed through application of symmetry group, which can be completed with construction given earlier. The coordinates of the points (Table 1) have been devised in terms of the following intuitive modeling parameters and joined with $2 \mathrm{D}$ entities.

a. n- Number of petals in the motif

b. R- Size of the motif defined by the radius of outer circle

c. r- Size of central circular part of the motif defined by the radius of inner circle
With the different combinations and using line or arc between the points one can draw different sense in the floral motifs. Here the variety of motifs can be produced with varying the value of Number of petals (n), radius (R) of outer circle, and the radius ( $r$ ) of inner circle. This geometrical design can produce the three motifs as shown in figure 3 .

Table 1: Coordinates of the Points of the Floral Motif's petal

\begin{tabular}{|c|c|}
\hline Points & Coordinates of Point \\
\hline $\mathrm{A}$ & $\mathrm{R}, 0$ \\
\hline $\mathrm{B}$ & $R \operatorname{Cos} \frac{2 \pi}{n}, R \operatorname{Sin} \frac{2 \pi}{n}$ \\
\hline $\mathrm{C}$ & $2 R \operatorname{Cos} \frac{\pi}{n} \operatorname{Sin} \frac{6 \pm n}{6 n} \pi, 2 R \operatorname{Sin} \frac{\pi}{n} \operatorname{Sin} \frac{6 \pm n}{6 n} \pi$ \\
\hline $\mathrm{I}$ & $r \operatorname{Cos} \frac{2 \pi}{n}, r \operatorname{Sin} \frac{2 \pi}{n}$ \\
\hline $\mathrm{J}$ & \\
\hline
\end{tabular}

Some other motifs/designs are being constructed with the number of counting squares. In such designs the innovative ideas of the designer precede towards the rangoli design motifs. The design of such motifs depends upon the creativity and practice of the designer. An array of lines in objective to constructing the squares provides the best platform for producing these motifs. A lined grid is used for initial construction and the dotted grid can be easily parameterized in the term of its sides [5]. From figure shown in table 2, "a" and " $b$ " represents the side OE and OP respectively than the coordinates of all points in terms of "a" and "b" are as given in table 2. In the same way, the different combination of lines creates different rangoli designs motifs as shown in figure 4 .

\section{GENERATION OF DESIGN PATTERNS}

The appeal of rangoli geometric design lies in its logical interrelation of the different motifs. There are one or more motifs in the traditional rangoli design patterns. Motifs are placed in a pattern with a special theme e.g. aligning motifs along a specified path or in a rectangular/polar array. 
Table 2: Coordinates of the Points of the Lined Grid

\begin{tabular}{|c|c|c|c|c|c|}
\hline Points & $\begin{array}{c}\text { Coordinates } \\
\text { of Point }\end{array}$ & Points & $\begin{array}{c}\text { Coordinates } \\
\text { of Point }\end{array}$ & Points & $\begin{array}{c}\text { Coordinates } \\
\text { of Point }\end{array}$ \\
\hline $\mathrm{A}$ & $0, \frac{a}{5}$ & $\mathrm{M}$ & $b, \frac{2 a}{5}$ & $\mathrm{Y}$ & $\frac{2 b}{5}, \frac{a}{5}$ \\
\hline $\mathrm{B}$ & $0, \frac{2 a}{5}$ & $\mathrm{~N}$ & $b, \frac{a}{5}$ & $\mathrm{Z}$ & $\frac{3 b}{5}, \frac{a}{5}$ \\
\hline $\mathrm{C}$ & $0, \frac{3 a}{5}$ & $\mathrm{O}$ & 0,0 & 0 & $\frac{4 b}{5}, \frac{a}{5}$ \\
\hline $\mathrm{D}$ & $0, \frac{4 a}{5}$ & $\mathrm{P}$ & $b, 0$ & 1 & $\frac{b}{5}, \frac{a}{5}$ \\
\hline $\mathrm{E}$ & $0, a$ & $\mathrm{Q}$ & $\frac{4 b}{5}, 0$ & 2 & $\frac{b}{5}, \frac{2 a}{5}$ \\
\hline $\mathrm{F}$ & $\frac{b}{5}, a$ & $\mathrm{R}$ & $\frac{3 b}{5}, 0$ & 3 & $\frac{b}{5}, \frac{3 a}{5}$ \\
\hline $\mathrm{G}$ & $\frac{2 b}{5}, a$ & $\mathrm{~S}$ & $\frac{2 b}{5}, 0$ & 4 & $\frac{b}{5}, \frac{4 a}{5}$ \\
\hline $\mathrm{H}$ & $\frac{3 b}{5}, a$ & $\mathrm{~T}$ & $\frac{b}{5}, 0$ & 5 & $\frac{2 b}{5}, \frac{4 a}{5}$ \\
\hline $\mathrm{I}$ & $\frac{4 b}{5}, a$ & $\mathrm{U}$ & $\frac{2 b}{5}, \frac{2 a}{5}$ & 6 & $\frac{3 b}{5}, \frac{4 a}{5}$ \\
\hline $\mathrm{J}$ & $b, a$ & $\mathrm{~V}$ & $\frac{2 b}{5}, \frac{3 a}{5}$ & 7 & $\frac{4 b}{5}, \frac{4 a}{5}$ \\
\hline $\mathrm{K}$ & $b, \frac{4 a}{5}$ & $\mathrm{~W}$ & $\frac{3 b}{5}, \frac{3 a}{5}$ & 8 & $\frac{4 b}{5}, \frac{3 a}{5}$ \\
\hline $\mathrm{L}$ & $b, \frac{3 a}{5}$ & $\mathrm{X}$ & $\frac{3 b}{5}, \frac{2 a}{5}$ & 9 & $\frac{4 b}{5}, \frac{2 a}{5}$ \\
\hline & $\mathrm{r}$ & & & \\
\hline
\end{tabular}

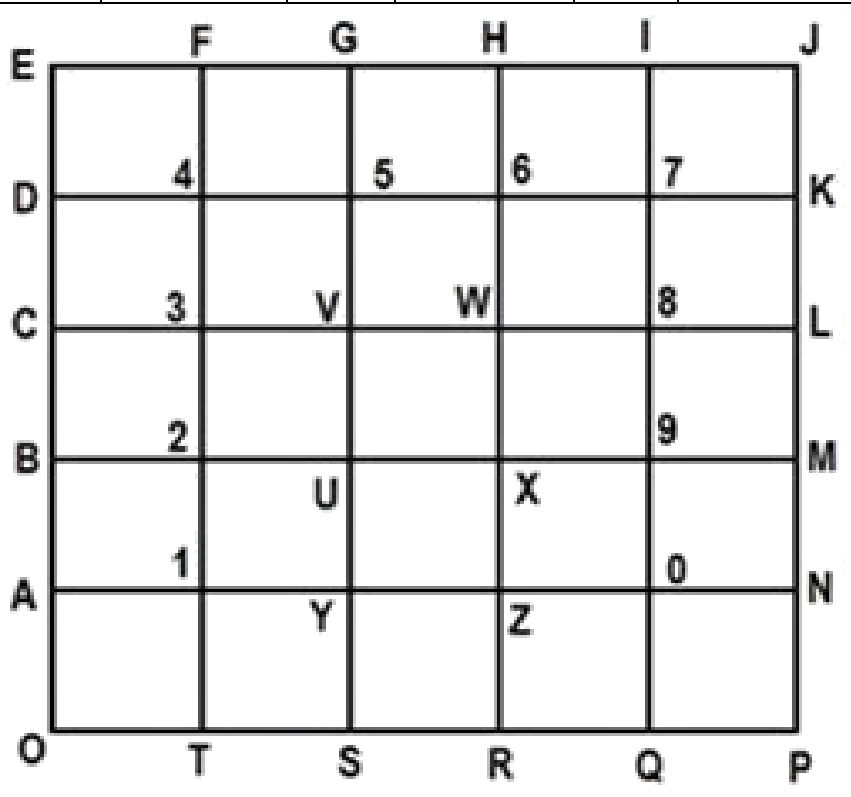

These rangoli design patterns are composed of motifs which are multiplied and prearranged in regular sequences by using transformations between them. The design patterns are generated in a particular sequence followed by hierarchy form low level entities like petal/motif to compound motif and then the pattern. The hierarchy level for pattern generation is as illustrated in figure 5. The role of transformations is to define positions of the motifs with respect to pattern in a $2 \mathrm{D}$ plane. Different traditional rangoli design patterns have been produced using this CAD tool (Figure 6).

\section{CONCLUDING REMARKS}

This work establishes a convergence of traditional rangoli designs and technology. The traditional skills of rangoli making and technologies can be engaged and the designs can also be digitized for storage and later use. In the area of digitizing cultural artifacts, this presented tool can also be used on other traditional planar artworks. This allows us actively to preserve the Indian cultural heritage. The rangoli design patterns can be generated by the presented tool. It can be easily interfaced with a variety of application programs and can produces computer understandable designs that support diverse integrated manufacturing process. It supports a variety of manufacturing applications such as robotic arm/machine $\mathrm{CNC}$ and laser cutter machines etc.

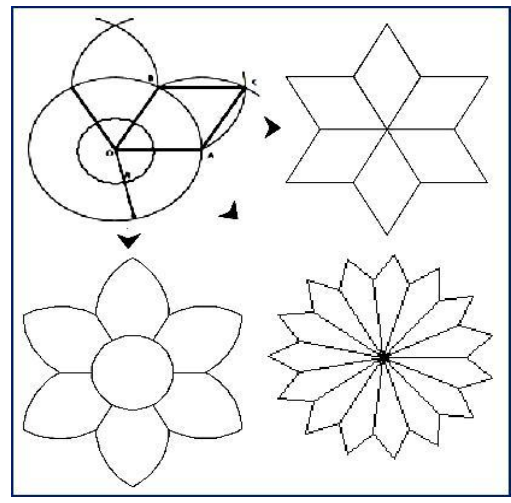

Fig. 3: Different Geometrical Design of Rangoli Floral Motifs

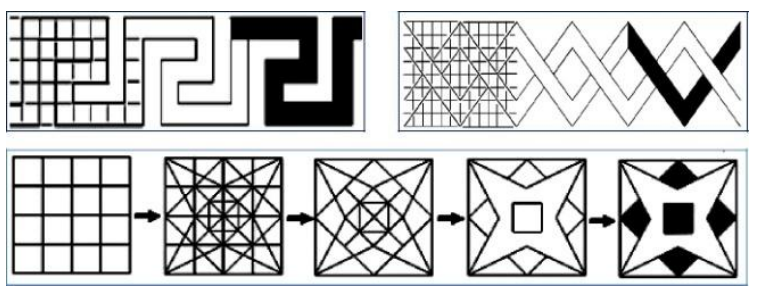

Fig. 4: Hierarchy level in Construction of different Rangoli Design Motifs from lined grid

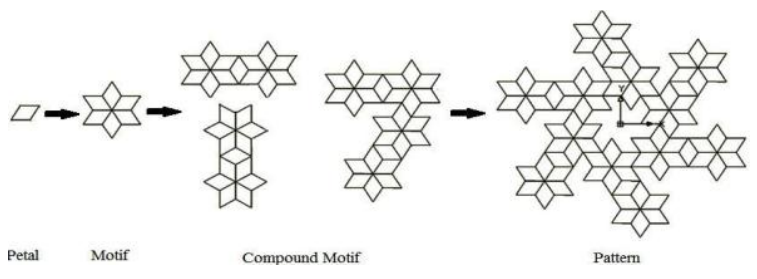

Fig. 5: Hierarchical Representation of Rangoli Design Patterns

\section{ACKNOWLEDGMENTS}

Authors acknowledge the immense help received from the scholars whose articles are cited and included in references of this paper. The authors are also grateful to authors / editors / publishers of all those articles, journals and Bookroom where the literature for this article has been reviewed and discussed. 

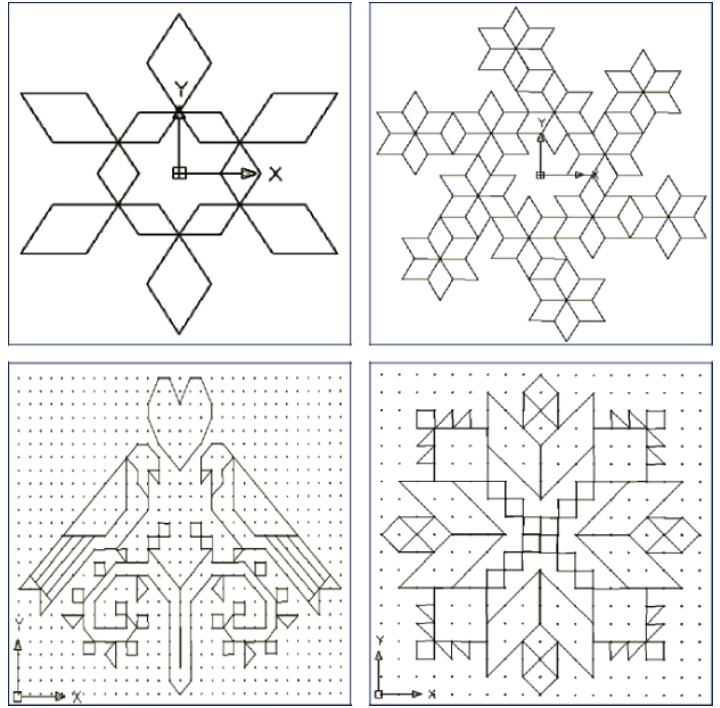

Fig. 6: Rangoli design patterns produced using the CAD tool

\section{REFERENCES}

[1] Cromwell, P. R. (2010). Islamic geometric designs from the Topkap1 Scroll I: unusual arrangements of stars. Journal of Mathematics and the Arts, 4(2), 73-85.

[2] Singh, K., Kumar, A., \& Sharma, N. (2013). An Innovative Approach to Recognize and Generate the Design Patterns as Formative Concepts to Support the Design Computation. International Journal of Computer Applications, 63(12), 15-19.
[3] Wong, M. T., Zongker, D. E., \& Salesin, D. H. (1998, July). Computer-generated floral ornament. In Proceedings of the 25th annual conference on Computer graphics and interactive techniques (pp. 423-434). ACM.

[4] Gulati, V., Singh, K., \& Katyal, P. (2012). A CAD Paradigm for Generating Woodworking Motifs. International Journal of Computer Applications, 47(5), $38-40$.

[5] Singh, K., Kumar, A., \& Sharma, N. (2013). The Mathematical Aspects in Art to Create Decorative Effect in Design Patterns. International Journal of Innovative Technology and Exploring Engineering, 2(2), 36-39

[6] Saksena, J. (1985). Mandana, a Folk Art of Rajasthan. New Delhi: Crafts Museum

[7] Dutta, S. (2011). The Mystery of Indian Floor Paintings. Chitrolekha International Magazine on Art and Design, 1(1), 14-27.

[8] Sethi, R. (1985). Aditi. The Living Arts of India. Smithsonian Institution. Wasnington DC.

[9] Huyler, S. P. (1994). Painted prayers: Women's art in village India. Thames and Hudson.

[10] Jones C. C. (2002). Rangoli: Elder Women Creating Sacred Geography. Tap Dancing Lizard, USA.

[11] Giesecke, F. E., Mitchell, A., Spencer, H. C., \& Merrell, C. (1958). Technical drawing. Macmillan.

[12] http://www.hennapage.com/henna/encyclopedia/rangoli/r angoliHPfree.pdf 